\title{
New Results in Chaotic Time-Reversed Electromagnetics: High Frequency One-Recording-Channel Time-Reversal Mirror
}

\author{
Steven M. Anlage ${ }^{a, b, c}$, J. Rodgers $^{d}$, S. Hemmady $^{a, b, c, e}$ \\ J. HART ${ }^{b, d}$, T.M. ANTONSEN ${ }^{b, c, d}$ AND E. OTT ${ }^{b, c, d}$ \\ ${ }^{a}$ Center for Nanophysics and Advanced Materials, Physics Department \\ University of Maryland, College Park, MD 20742-4111, USA \\ ${ }^{b}$ Physics Department, University of Maryland \\ College Park, MD 20742-4111, USA \\ ${ }^{c}$ Department of Electrical and Computer Engineering \\ University of Maryland, College Park, MD 20742, USA \\ ${ }^{d}$ Institute for Research in Electronics and Applied Physics \\ University of Maryland, College Park, MD 20742-3511, USA \\ ${ }^{e}$ Malibu Research Associates Inc. \\ 3760A Calle Tecate, Camarillo CA 93012-5059, USA
}

We experimentally demonstrate a new electromagnetic one-recording-channel time-reversal mirror that can operate at high frequencies and high bandwidths. The experiments are carried out in a $1 \mathrm{~m}^{3}$ ray-chaotic enclosure using two simple antennas. The input is a $7.0 \mathrm{GHz}$ signal that is amplitude modulated with a $60 \mathrm{~ns}$ long pulse. The time-reversal focused signal has a peak-signal-to-noise ratio of about $9 \mathrm{~dB}$, and is very sensitive to small perturbations to the ray-chaotic enclosure. The results are consistent with expectations for single-recording-channel time-reversal mirrors, and establish a new platform for study of fundamental issues in time-domain wave chaos, as well as novel applications.

PACS numbers: 03.50.De, 05.45.Mt, 41.20.Jb, 42.65.Hw

\section{Introduction}

Time-reversal symmetry of the wave equation allows one to examine several unique phenomena associated with wave physics. One of these phenomena is the time-reversal mirror (TRM). If all of the wave excitations in a system can be captured on a closed surface, and if they can be time-reversed and re-injected into 
the system, then the wave excitations will undo any phase changes and distortions that they suffered in time-forward propagation [1]. The TRM can be used to focus waves back to their point of origin, or to create excitations that converge on scattering centers located in an inhomogeneous medium [2]. When used in conjunction with a time-reversed excitation (sink), super-resolution focusing of the time-reversed signals can be achieved [3, 4].

The TRM and its applications are greatly simplified in the presence of multiple scattering. In particular, ray-chaotic enclosures allow one to simplify the TRM to a single source/receiver $[5,6]$ and still obtain very good time-reversed characteristics. Such wave chaotic TRMs have been demonstrated in acoustic systems. The electromagnetic TRMs have been demonstrated at relatively low frequency $(2.45 \mathrm{GHz})$ with relatively small bandwidth $(150 \mathrm{MHz})$ using multiple antennas $[7,8]$. Here we present results on a new type of electromagnetic TRM that can operate at higher frequencies (demonstrated at $7 \mathrm{GHz}$ in this case) and high bandwidth $(2 \mathrm{GHz})$ utilizing a single-recording-channel antenna.

\section{Experiment}

The one-recording-channel TRM experiment was carried out in a ray-chaotic metallic box of exterior dimensions $127 \times 127 \times 66 \mathrm{~cm}^{3}$, with a volume of about $1 \mathrm{~m}^{3}$. Inside this box there are two metallic mode-stirring paddles, similar in design to those used in electromagnetic mode-stirred chambers. The presence of these paddles insures the existence of ray chaos inside the enclosure. Two antennas (Hertzian dipoles) are used in the experiment. They are located on two adjacent walls of the enclosure and consist of short straight segments of the center conductor of a coaxial connector projecting $\approx 1 \mathrm{~cm}$ into the enclosure (see Fig. 1).

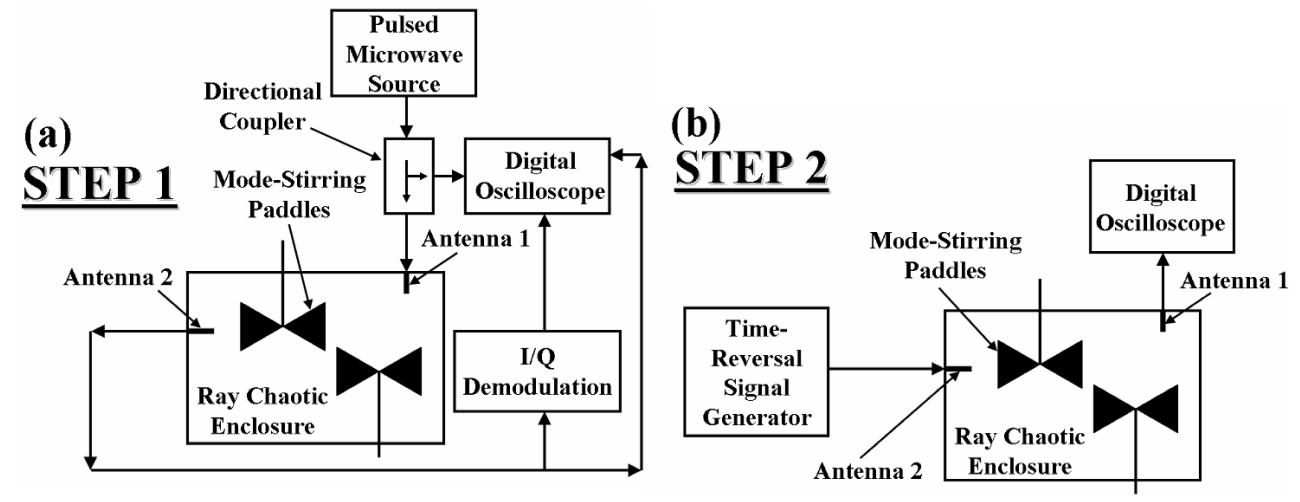

Fig. 1. Schematic diagram of the two-step high frequency electromagnetic one-recording-channel time-reversal mirror experiment. (a) Pulsed excitation is injected into the ray-chaotic enclosure and both the input and output signals are recorded. (b) The time-reversed signal from step 1 is applied to antenna 2 and the focused response is measured at antenna 1 with the oscilloscope. 
The TRM was demonstrated in a 2-step process (refer to Fig. 1). First, a pulse of microwaves was injected into the ray-chaotic enclosure. A $7.0 \mathrm{GHz}$ carrier signal was used and it was amplitude modulated with a 60 ns long pulse. This signal was broadcasted into the enclosure through antenna 1 (Fig. 1a). The transmitted signal (coda) at antenna 2 was recorded on a digital oscilloscope for one orientation of the paddles. In the second step, the recorded $I / Q$ coda was time-reversed on a computer and sent to a vector signal generator which created a time-reversed coda by modulating a $7.0 \mathrm{GHz}$ carrier signal. This signal was applied to antenna 2 of the ray-chaotic enclosure (Fig. 1b), where the paddles were in the same orientation as in step 1. The time-reversed signal then propagated through the enclosure, and the signal appearing at antenna 1 was collected and recorded in a digital oscilloscope.

\section{Results}

Figure 2a shows a recording of the input pulse sent to antenna 1, over a $400 \mathrm{~ns}$ timescale. This $60 \mathrm{~ns}$ long pulse was measured through a directional coupler attached to antenna 1 . Figure $2 \mathrm{~b}$ shows the output wave form recorded at antenna 2 over a $4 \mu$ s timescale, demonstrating the duration and complexity of a part of the coda response. This $4 \mu \mathrm{s}$ long coda was used in the time-reversal experiment.
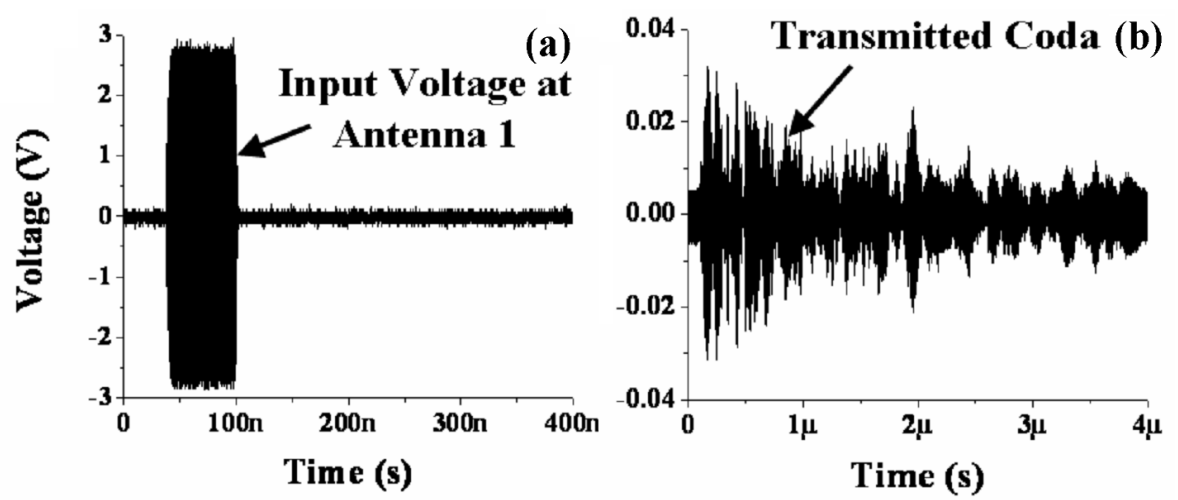

Fig. 2. (a) Measured input pulse going in to antenna 1, consisting of a $60 \mathrm{~ns}$ long modulation of a $7.0 \mathrm{GHz}$ carrier frequency signal at $+24 \mathrm{dBm}$. (b) Transmitted signal coda measured at antenna 2 . Let us note the factor of 10 difference in timescales.

Figure 3 shows the reconstructed pulse measured at antenna 1 after the time-reversed coda had been injected into antenna 2. Let us note that the reconstructed pulse occurs near the end of this $4 \mu$ s long time window, as expected. Upon close examination it was found that the reconstructed pulse was also $60 \mathrm{~ns}$ long, exactly like the incident pulse. It is clear from this figure that a significant amount of 


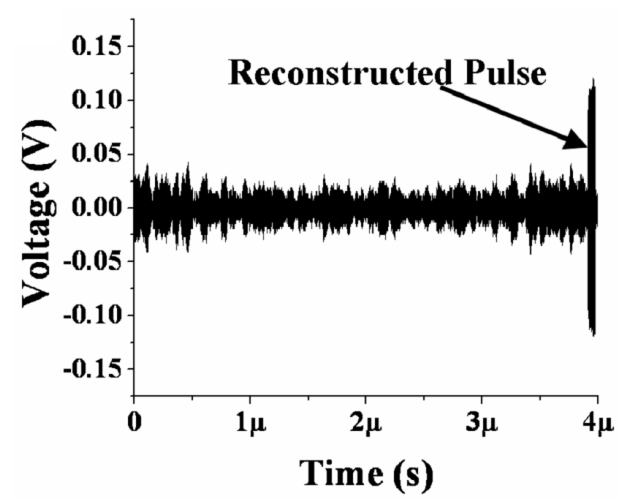

Fig. 3. Measurement of signal at antenna 1 due to the injection of a $4 \mu$ s long time-reversed coda signal at antenna 2. A $60 \mathrm{~ns}$ long pulse appears near the end of this $4 \mu \mathrm{s}$ long window.
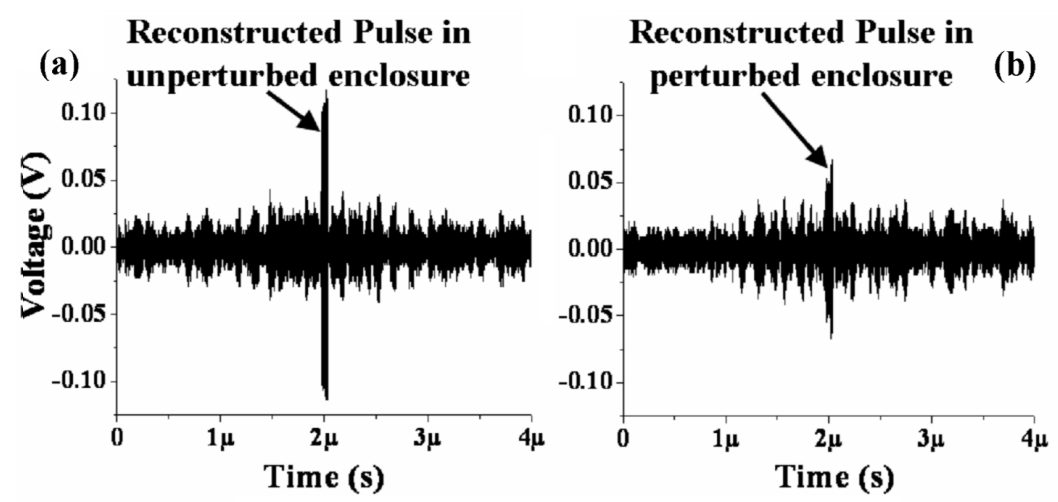

Fig. 4. Reconstructed pulses measured at antenna 1 of the ray-chaotic enclosure after a $4.0 \mu$ s long time-reversed signal was injected at antenna 2. (a) Pulse reconstructed in an unperturbed enclosure, and (b) pulse reconstructed after a small perturbation to one wall of the enclosure. Let us note that both plots are on the same scales.

noise accompanies the reconstructed pulse, compared to the input pulse shown in Fig. 2a.

Figure $4 \mathrm{a}$ shows the reconstructed pulse, now centered in a $4 \mu$ s wide window. To test the sensitivity of the reconstruction to a small perturbation of the raychaotic enclosure, the following procedure was followed. One finger was pressed against one wall of the enclosure. A small displacement of that wall (perhaps $1 \mathrm{~mm}$ or so) was created. The reconstructed pulse was then measured under this perturbed condition, and the result is shown in Fig. 4b. Let us note that the amplitude of the reconstructed 60 ns long pulse is reduced, and the "noise" on either side of the pulse is enhanced, resulting in a reduced signal-to-noise ratio (SNR). A quantitative measure of SNR was made by taking the ratio of peak voltage 
value during the reconstructed pulse to that value in the wave form away from the pulse ( which we call peak-SNR; peak-SNR $=20 \log _{10}\left(V_{\text {signal,peak }} / V_{\text {noise,peak }}\right)$ ). The unperturbed cavity reconstruction has a peak-SNR of $8.7 \mathrm{~dB}$, while that in the perturbed case is reduced to $4.7 \mathrm{~dB}$. (The measured incident pulse had a peak-SNR of about $23 \mathrm{~dB}$.) This demonstrates the sensitivity of a relatively simple one-recording-channel time-reversal mirror to very small perturbations.

\section{Discussion}

The reconstructed pulse shown in Figs. 3 and 4 is not a perfect replica of the incident pulse shown in Fig. 2. In principle all of the signal injected into the enclosure must be captured and time-reversed to create a perfect replica. In practice there are three reasons that a perfect reconstruction is not obtained in this experiment. These are:

i) use of a finite time window,

ii) loss of information from the use of a single recording channel,

iii) resistive losses in the system.

Concerning the effect of (i), it was shown in [6] that the quality of the time-reversal focusing is dependent on the size of the time-reversal window. In this case a $4 \mu$ s long window is not sufficient to capture all of the coda wave dynamics required to reconstruct the pulse. One should at least enter the limit where the time reversal window width greatly exceeds the inverse of the mean spacing between modes in the frequency domain, but in this case they are in fact about equal.

Concerning the effect of (ii), in the ideal case of zero losses (i.e., the absence of effect (iii)) and an infinite time window (i.e., the absence of effect (i)), perfect time reversal would be obtained if the signals propagating out of all the ports (i.e., both ports 1 and 2) were reversed. However, in our experiment we only do this at port 2 .

Finally, with respect to the effect of (iii), it should also be noted that the electromagnetic enclosure has losses associated with finite conductivity of the walls. The loss parameter of the cavity $(\alpha)$ is defined as the ratio of the typical $3 \mathrm{~dB}$ bandwidth of the resonance modes to the mean spacing between eigenfrequencies [9]. The enclosure has a loss parameter $\alpha \approx 1.2$ in this case, meaning that the modes are entering the overlapping limit. This also results in loss of information and a degradation of the reconstructed pulse. $S$-parameter measurements of the enclosure show that $1-\left|S_{11}\right|^{2} \approx-3 \mathrm{~dB}$ and $\left|S_{21}\right|^{2} \approx-25 \mathrm{~dB}$ near $7 \mathrm{GHz}$ (although both are strongly fluctuating with frequency). Because both antennas are roughly identical, these results mean that very little signal re-emerges from the cavity after it is excited, either through antenna 1 or antenna 2 . The majority of the $3 \mathrm{~dB}$ return loss is promptly reflected at antenna 1 due to the antenna impedance mismatch [10-12]. This means that roughly half the injected pulse energy actually enters the cavity, and only a fraction of a percent of all the energy is captured 
at antenna 2. In spite of this very large loss, we still observe (Fig. 4) good pulse reconstruction (although the energy in the reconstructed pulse is only a small fraction of the energy supplied in the re-injected time-reversed signal).

\section{Conclusions}

We have demonstrated a new high frequency electromagnetic one-recording-channel time-reversal mirror that takes advantage of a ray-chaotic enclosure. The results are consistent with expectations and estimates for a wave chaotic TRM. Our TRM can operate at frequencies up to $20 \mathrm{GHz}$ and with bandwidths of up to $2 \mathrm{GHz}$, making it possible to perform very high quality time-reversal focusing.

\section{Acknowledgments}

We thank Florian Schäfer for helpful discussions and Chris Bertrand for technical assistance. This work is supported by an ONR MURI entitled "Exploiting Nonlinear Dynamics for Novel Sensor Networks", grant N000140710734, an ONR DURIP grant N000140710708, and AFOSR under grant FA95500710049.

\section{References}

[1] M. Fink, D. Cassereau, A. Derode, C. Prada, P. Roux, M. Tanter, J.-L. Thomas, F. Wu, Rep. Prog. Phys. 63, 1933 (2000).

[2] C. Prada, F. Wu, M. Fink, J. Acoust. Soc. Am. 90, 1119 (1991).

[3] J. de Rosny, M. Fink, Phys. Rev. Lett. 89, 124301 (2002).

[4] R. Carminati, J.J. Saenz, J.-J. Greffet, M. Nieto-Vesperinas, Phys. Rev. A 62, $012712(2000)$.

[5] C. Draeger, M. Fink, Phys. Rev. Lett. 79, 407 (1997).

[6] C. Draeger, M. Fink, J. Acoust. Soc. Am. 105, 611 (1999).

[7] G. Lerosey, J. de Rosny, A. Tourin, A. Derode, G. Montaldo, M. Fink, Phys. Rev. Lett. 92, 193904 (2004).

[8] G. Lerosey, J. de Rosny, A. Tourin, A. Derode, M. Fink, Appl. Phys. Lett. 88, 154101 (2006).

[9] S. Hemmady, X. Zheng, E. Ott, T.M. Antonsen, S.M. Anlage, Phys. Rev. Lett. 94, 014102 (2005).

[10] S. Hemmady, X. Zheng, T.M. Antonsen, E. Ott, S.M. Anlage, Phys. Rev. E 71, 056215 (2005).

[11] X. Zheng, T.M. Antonsen Jr., E. Ott, Electromagnetics 26, 3 (2006).

[12] X. Zheng, T.M. Antonsen Jr., E. Ott, Electromagnetics 26, 37 (2006). 